\title{
Use of Adsorbents for Heat and Mass Transfer Applications
}

\author{
F. Handan Tezel \\ University of Ottawa, Canada \\ 161 Louis Pasteur, Ottawa, ON K1N 6N5 \\ handan.tezel@uottawa.ca
}

This talk will give some examples of use of adsorbents in mass and heat transfer applications. The application of adsorbents for adsorption separation processes for gas and liquid systems are well known. This talk will give examples of adsorption separation processes where adsorbents are used, as well as some examples of membrane processes and thermal energy storage systems where adsorbents are used. After a brief introduction to adsorbents and adsorption processes, their application in air and water pollution control, renewable fuels production, adsorbent membranes, and thermal energy storage for solar and waste heat applications will be discussed. Examples will include removal of greenhouse gas carbon dioxide and its conversion to synthetic fuel, production of natural gas from landfill gas, production of bio-alcohols from agricultural biproducts and using adsorption for storing solar and waste thermal energy. 